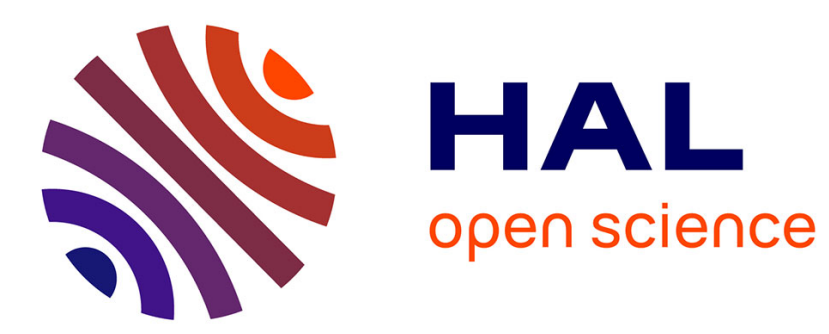

\title{
Incidences économiques, politiques et redistributives des réformes des retraites en Europe
}

Michel Aglietta, Jean Chateau, Jacky Fayolle, Michel Juillard, Jacques Le

Cacheux, Gilles Le Garrec, Vincent Touzé

\section{- To cite this version:}

Michel Aglietta, Jean Chateau, Jacky Fayolle, Michel Juillard, Jacques Le Cacheux, et al.. Incidences économiques, politiques et redistributives des réformes des retraites en Europe: Une exploration avec le modèle INGENUE. Revue Economique, 2002, 53 (4), pp.787 - 808. 10.3917/reco.534.0787 . hal03384673

\section{HAL Id: hal-03384673 \\ https://hal.science/hal-03384673}

Submitted on 19 Oct 2021

HAL is a multi-disciplinary open access archive for the deposit and dissemination of scientific research documents, whether they are published or not. The documents may come from teaching and research institutions in France or abroad, or from public or private research centers.
L'archive ouverte pluridisciplinaire HAL, est destinée au dépôt et à la diffusion de documents scientifiques de niveau recherche, publiés ou non, émanant des établissements d'enseignement et de recherche français ou étrangers, des laboratoires publics ou privés. 


\title{
Incidences économiques, politiques et redistributives des réformes des retraites en Europe
}

\author{
Une exploration avec le modèle INGENUE
}

\author{
Équipe INGENUE*
}

\begin{abstract}
Cet article présente trois simulations de l'impact de réformes des systèmes de retraite en Europe. Ces simulations sont réalisées avec le modèle INGENUE, modèle d'équilibre général calculable à générations imbriquées de l'économie mondiale. Les réformes envisagées sont des variantes autour d'un compte central qui postule le maintien d'un taux constant de remplacement des pensions sur les salaires nets. Les trois réformes étudiées sont : le maintien d'un taux constant de cotisation sociale, le maintien d'un taux constant de remplacement des pensions sur le salaire brut, et l'allongement de la durée d'activité. Les impacts de ces réformes sont alors examinés sous les angles économique, politique et redistributif.
\end{abstract}

\section{THE DISTRIBUTIVE ALLOCATIVE AND POLICY EFFECTS OF EUROPEAN PUBLIC PENSIONS REFORMS \\ AN INVESTIGATION WITH THE INGENUE MODEL}

This paper presents various effects of three simulated reforms of the European pay-as-you-go system. These are investigated with INGENUE: a multi-region, general equilibrium, overlapping-generation model of the world economy. Reforms are presented around a baseline scenario where constant replacement ratio of public pension over net wage rate is assumed. Alternative reforms are: constant contribution rate, legal retirement age postponement and constant replacement ratio of public pension over gross wage rate. We then investigate the allocative, distributive and politic effects of these various policy.

Classification JEL : C68, F4, H55, D63

* L'équipe INGENUE est actuellement composée de Michel Aglietta (Université Paris $\mathrm{X}$-Nanterre, Institut universitaire de France, et CEPII), Jean Chateau (CEPII), Jacky Fayolle (OFCE et Université Pierre Mendès-France de Grenoble), Michel Juillard (Université Paris VII et CEPREMAP), Jacques Le Cacheux (Université de Pau et de Pays de l'Adour et OFCE), Gilles Le Garrec (Université Paris I-Panthéon-Sorbonne) et Vincent Touzé (OFCE, THEMA et Institut d'études politiques de Lille). Outre le CEPII, le CEPREMAP et l'OFCE, co-développeurs du modèle INGENUE, le projet a bénéficié du soutien financier de l'Institut Caisse des dépôts et du Conseil national du crédit et des titres. Les travaux issus de ce modèle ont été présentés dans de nombreux séminaires et conférences, où ils ont bénéficié des commentaires critiques des participants. Les auteurs tiennent à remercier un rapporteur de la revue pour ces précieux commentaires. 


\section{INTRODUCTION}

Le vieillissement tendanciel de la population européenne, combiné à l'entrée en retraite des générations du baby-boom, semble devoir pousser inéluctablement à l'adoption de réformes des systèmes publics de retraite, soit sous la pression d'un électorat majoritaire mécontent, soit du fait d'une éventuelle injustice dans le traitement de certaines générations.

En matière de finances sociales, deux stratégies polaires sont envisageables : la législation sur les recettes est invariante (les taux de cotisation sociale sont maintenus constants) ou la législation sur les dépenses est inchangée (le mode de calcul des pensions retraite reste fixe : un taux de remplacement constant sur le salaire brut est garanti). Une solution intermédiaire consiste à adopter un mélange de ces deux stratégies : il s'agit de la politique de maintien d'un taux de remplacement constant sur le salaire net, c'est-à-dire le revenu du travail dont sont soustraites les cotisations sociales. Cela signifie que les taux de cotisation sociale et de remplacement sur le salaire brut deviennent tous les deux variables. Cette évolution paraît des plus crédibles au regard des recommandations qui émanent des études réalisées dans de nombreux pays européens. Ainsi, en France, une telle proposition est apparue dans le cadre des travaux de la mission Retraite $^{1}$ (cf. Cottave [1991]) : il faut chercher à maintenir une " parité entre les revenus des pensions et les revenus des salariés ». Les deux autres scénarios apparaissent donc comme des solutions extrêmes qui encadrent la solution «maintien d'une parité de revenu ». Une troisième voie de réforme est souvent évoquée : il s'agit de l'allongement de la durée d'activitée ${ }^{2}$, qui présente le double avantage de réduire la durée de versement des pensions tout en augmentant la durée de paiement des cotisations. Une telle réforme permettrait donc une baisse du taux de cotisation sans recourir à une réduction du montant des pensions. Bien entendu, une telle mesure présuppose qu'il n'existe pas de problème d'employabilité des travailleurs âgés, et que le coût moral de la réforme (caractère pénible d'un travail, réduction du loisir) est largement compensé par l'augmentation résultante de revenu.

Dans cet article, nous étudions, à l'aide du modèle de l'économie mondiale INGENUE, modèle d'équilibre général calculable à générations imbriquées, l'impact de ces quatre possibilités d'évolution de la législation des finances sociales en Europe. La stratégie «maintien d'une parité entre les revenus d'activités et les pensions » est considérée comme le scénario de référence. Les impacts des différentes réformes sont examinés sous les angles économique (quelles sont les incidences dans la zone Europe, sur le marché mondial du capital et dans le reste du monde ?), politique (sont-elles politiquement via-

1. Travaux réalisés à la demande de Michel Rocard, Premier ministre de l'époque.

2. En France, une telle mesure a été adoptée pour le régime général de retraite du secteur privé en 1994 par le gouvernement Balladur. Par ailleurs, cette réforme a pérennisé une indexation des pensions sur les prix à la consommation moins favorable aux retraités que l'indexation sur le salaire net : à la différence de cette dernière, en effet, à long terme, les retraités ne bénéficient plus de la croissance et le taux de remplacement net des pensions baisse tendanciellement. 
bles ?) et redistributif (quelles sont les générations qui y gagnent, qui y perdent ? Dans quelle mesure y a-t-il une plus grande justice entre les générations ?).

Ces différents points de l'étude sont répartis dans trois sections. La première section résume les hypothèses sur lesquelles a été construit le modèle INGENUE, ainsi que les réformes étudiées. La deuxième section explore le volet macroéconomique de l'incidence des réformes : évolution du taux d'épargne, variation du taux d'intérêt mondial, etc. Enfin, la dernière section présente une analyse politique et redistributive des réformes. Pour construire un solde politique, on identifie, grâce à l'évolution du bien-être de chaque génération, celles qui y gagnent et celles qui y perdent. Un critère financier de taux de rendement interne du système par répartition est également calculé. Enfin, un critère de planificateur est utilisé pour comprendre l'incidence des réformes dans une perspective d'optimum social.

\section{PRÉSENTATION SYNTHÉTIQUE DU MODĖLE ET DU SCÉNARIO CENTRAL}

\section{Le modèle INGENUE}

INGENUE est un modèle d'équilibre général calculable à générations imbriquées dans la lignée de celui d'Auerbach et Kotlikoff ${ }^{1}$ [1987], mais étendant le cadre analytique à l'économie mondiale. Le monde y est divisé en six grandes zones selon des critères démographiques et socio-économiques ${ }^{2}:$ trois zones économiquement développées (Europe de l'Ouest, Amérique du Nord et Japon) pour lesquelles la transition démographique est déjà avancée, et trois zones en développement. Parmi celles-ci, l'une est déjà très engagée dans la transition démographique (zone SV comprenant notamment l'Asie de l'Est dont la Chine, la Russie...); une autre a amorcé la baisse de sa fécondité (zone $J 1$ : Amérique du Sud, monde indien,...); enfin, dans la dernière région, la transition démographique n'est actuellement que faiblement perceptible (zone J2: Afrique, Amérique centrale, Asie centrale...).

Le modèle repose sur trois hypothèses principales issues de la théorie standard de l'équilibre général intertemporel. La première est la relation entre structure par âge et épargne domestique qui découle de la conjonction de la théorie du cycle de vie (Modigliani [1986]) et de la coexistence à chaque date de générations différentes d'individus (Diamond [1965]) ${ }^{3}$. Ensuite, dans la tradition des modèles de croissance développés à la suite de Solow [1956], un bien unique composite est produit dans chaque région au moyen d'une fonction de production "néoclassique » combinant deux facteurs de production «privatifs », le stock de capital au sens large installé dans la région et la population

1. On peut se reporter à Le Cacheux et Touzé [2002] pour une évaluation critique de cette littérature.

2. On pourra trouver des détails dans INGENUE [2001b].

3. Concrètement le pas du modèle étant quinquennal, quinze générations d'individus économiques autonomes et quatre générations d'enfants co-existent. 


\section{La structure du modele INGENUE}

À chaque période quinquennale, chacune des six zones $i$ est peuplée de quatre cohortes d'enfants, âgés de 0 à 19 ans, qui ne prennent aucune décision économique et sont à la charge de leurs parents, le coût de leur consommation étant supposé proportionnel à celle de leurs parents ; et de quinze cohortes d'adultes, âgées de 20 à 94 ans. Les naissances sont réparties parmi les ménages âgés de 20 à 39 ans en fonction des caractéristiques observées dans les différentes zones. Pour représenter simplement le processus d'allongement progressif de la vie, chaque cohorte est divisée en six à huit sous-cohortes, selon les zones, en fonction de l'âge de l'individu au moment de son décès : ceux qui meurent entre 55 et 59 ans, ceux qui meurent entre 60 et 64 ans, etc. Les proportions d'individus de chaque cohorte dans chacune des sous-cohortes d'espérance de vie différente sont ensuite choisies de manière à reproduire les évolutions projetées de la mortalité par âge dans les projections démographiques de l'onU.

Les ménages adultes ont des anticipations parfaites des taux de salaire et des taux d'intérêt réels futurs et connaissent notamment avec certitude la date de leur mort. Ils choisissent leur consommation et leur épargne de chaque période quinquennale en début de vie adulte avec l'objectif de maximiser une fonction d'utilité intertemporelle standard. Dans chaque région $i$, le problème de maximisation d'un individu de type de longévité $m=1, \ldots, \mathbf{M}^{i}$, dont la date d'entrée sur le marché du travail est $t$, est formalisé comme suit :

$\operatorname{Max} U_{t}^{i}=\sum_{g=1}^{s_{\text {max } / m}}\left(1+\rho^{i}\right)^{1-g} u\left(c_{g / m, t+g+1}^{i}\right) \quad i=1, \ldots, 6$ et avec $u(c)=\frac{c^{1-\sigma}}{1-\sigma}$

sous les contraintes

$$
\mu_{g / t+g}^{i} c_{g / m, z+g}^{i}=\omega_{t+g}^{i}+\mathbf{R}_{t+g-1} s_{g / m, t+g}^{i}-s_{g / m, t+g}^{i} g=1, \ldots, g_{\mathrm{max} / m}^{i},
$$

où

$\sigma \neq 1$ est identique dans toutes les zones;

$\rho^{i}$ est le taux de préférence pour le présent;

$\tau_{g / t+g}^{i}$ est un facteur d'échelle qui prend en compte le coût d'éducation des enfants ;

$c_{g / m, t+g}^{i}$ est la consommation à l'âge $g$;

$\omega_{t+g}^{i}=\lambda_{g} \cdot\left(1-\tau_{t}^{i}\right) w_{t+g}^{i}+\mathrm{P}_{g, t+g}^{i}$ est le revenu net après transferts $\left(\mathrm{P}_{g}^{i}\right)$ et cotisations sociales pour la retraite $\tau^{2} \cdot w^{2}$ avec $\left(\lambda_{g}=1, \mathrm{P}_{g}=0\right)$ si l'individu est actif et $\left(\lambda_{g}=0, \mathrm{P}_{g}>0\right)$ sinon;

$w^{i}$ est le salaire et $\mathrm{R}$ le facteur d'intérêt;

$s_{g / m, t+g}^{i}$ est le stock d'épargne à l'âge $g$.

Un bien unique est produit par des entreprises situées dans les différentes zones et employant la main-d'œuvre locale en combinaison avec du capital productif. Les entreprises ne supportent aucun coût d'ajustement et opèrent sur des marchés parfaitement concurrentiels, de sorte que les profits à l'équilibre sont nuls. Un taux constant de dépréciation $\delta$ affecte le stock de capital productif. La technologie est représentée par une fonction de production Cobb-Douglas, de la forme suivante (en omettant les indices temporels):

$f\left(k^{i}\right)=\mathrm{A}^{i}\left(k^{i}\right)^{\alpha}$ où $k^{i}=\frac{\mathrm{K}^{i}}{\mathrm{~L}^{i}}$ est l'intensité capitalistique, $\mathrm{K}^{i}$, le stock de capital productif et $\mathrm{L}^{i}$, la taille de la population active. 
Un progrès technique exogène fait crô̂tre le facteur d'échelle de la production $\mathrm{A}^{i}$ à un taux constant dans le temps. On suppose que ce rythme est dicté par la croissance de la productivité globale des facteurs du pays leader (États-Unis), les autres zones bénéficiant d'un rattrapage progressif, dont les caractéristiques sont également exogènes, obéissant à la loi de diffusion mondiale de la technologie suivante :

$$
\frac{\mathrm{A}_{t}^{i}}{\mathrm{~A}_{t-1}^{i}}=\left[1+\lambda^{t}\right] \frac{\mathrm{A}_{t}^{\text {Amerique }}}{\mathrm{A}_{t-1}^{\mathrm{Am} \text { r rique }}}\left[\beta^{\prime}+\left(1-\beta^{t}\right) \frac{\mathrm{A}_{t-1}^{\text {Amerique }}}{\mathrm{A}_{t-1}^{i}-1}\right],
$$

$\lambda$ est un accélérateur à la convergence en taux de croissance et $\beta$ est un frein à la convergence en niveau.

La solution du programme de maximisation du profit par les entreprises donne les demandes de facteurs de production, capital et travail, tandis que la confrontation, sur les marchés correspondants, aux offres de facteurs détermine les taux de salaire réels par zone et le taux d'intérêt réel mondial.

Dans chaque zone, le secteur public se résume à une caisse de retraite par répartition qui prélève, sur les actifs, des cotisations proportionnelles à leur salaire courant, verse aux retraités, inactifs au-delà de l'âge légal de départ à la retraite, des pensions, et équilibre ses comptes à chaque période. Toutes les zones sont censées avoir un tel système, dont les règles demeurent indéfiniment inchangées. Chaque système régional est caractérisé par deux paramètres institutionnels : l'âge légal (et impératif) de cessation d'activité, d'une part ; le taux de remplacement net, d'autre part. Le taux de cotisation est supposé endogène et varie à chaque période pour équilibrer la caisse de retraite.

L'accent étant mis, dans cette analyse, sur les transferts internationaux d'épargne entre les différentes zones, on peut résumer les interactions des variables macroéconomiques et démographiques en présence et leur résultante à l'aide de l'expression suivante, qui décrit le besoin de financement net de chaque région, donc la configuration des balances courantes :

Besoin net de capitaux de la région $i$ :

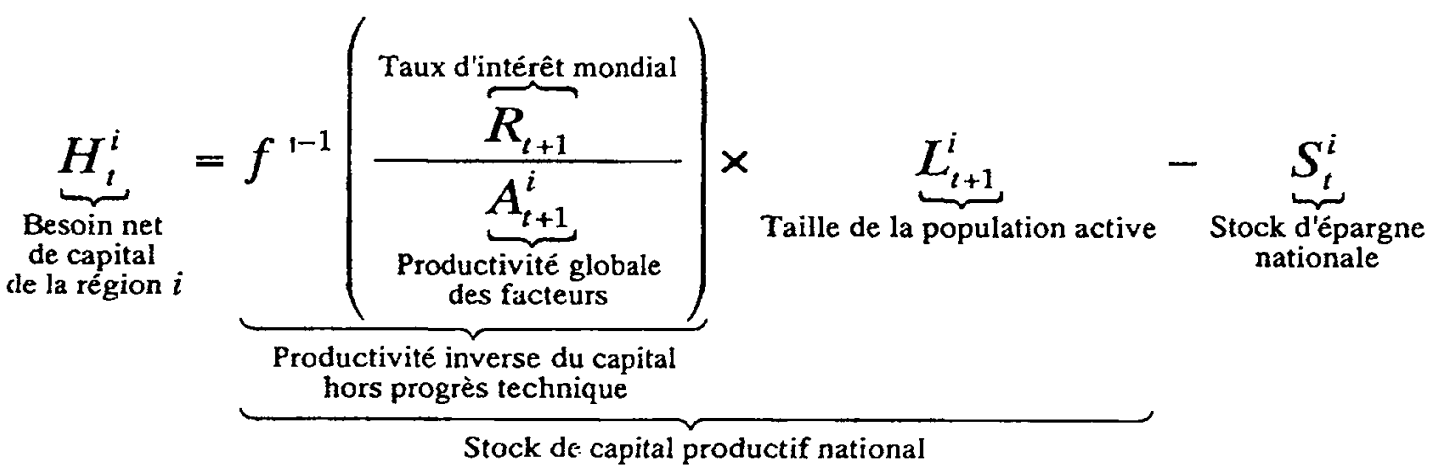

où $\mathrm{A}^{i} f^{\prime}\left(k^{i}\right)=\alpha \mathrm{A}^{i}\left(k^{i}\right)^{\alpha-1}$ est la productivité marginale du capital dans la région $i$. Balance courante de la région $i: \mathrm{B}_{t}^{i}=-\Delta \mathrm{H}_{t}^{i}$

Équilibre général de l'économie mondiale : $\sum_{i=1}^{6} \mathrm{H}_{i}^{i}=0$

Pour le calibrage du modèle, on peut se reporter à INGENUE (2001a). 
active, avec un facteur exogène de progrès technique (spécifique à chaque région). Enfin, l'échange mondial s'opère via le marché mondial du bien et le marché mondial du capital, supposés parfaits. Le libre transfert de capital entre les différentes régions du monde qui en découle aboutit à la formation d'un taux d'intérêt mondial unique, qui permettra notamment de déterminer les positions créancières et débitrices nettes entre les régions (Buiter [1981]).

\section{Une trajectoire de l'économie mondiale au $x \times\left.\right|^{\mathbf{e}}$ siècle : le scénario central}

Conséquence directe des hypothèses précédentes, la simulation du modèle fait apparaître l'échange intertemporel mondial comme mutuellement avantageux, et ce en raison du contraste des situations démographiques entre régions au cours du siècle. Ainsi, durant la première moitié du $\mathrm{XXI}^{\mathrm{e}}$ siècle, les pays riches et vieillissant par le haut de la pyramide des âges, combinant forte intensité capitalistique et population active déclinante, vont transférer de l'épargne dans des pays vieillissant par le bas de la pyramide, où l'intensité capitalistique est faible et la population active jeune et croissante. Dans ce cas, en effet, le taux de rendement du capital de ce monde financièrement intégré se trouve entre les taux de rendement des zones polarisées en situation d'autarcie financière. Transférer de l'épargne dans des activités productives à haut rendement peut maintenir le taux d'intérêt en condition d'efficience dynamique, tout en élevant la croissance mondiale et donc augmentant les revenus des zones débitrices, ce qui les met en position d'épargner et garantit la solvabilité de leurs emprunts.

Cette dynamique ne repose toutefois pas uniquement sur les seuls décalages dans les processus de transition démographique. Elle dépend aussi d'une conjecture sur le régime de croissance mondiale : ici on postule un rattrapage technologique qui, quoique généralisé, reste extrêmement lent ${ }^{1}$. Les hypothèses institutionnelles établissent une troisième source de différences entre les régions. Le scénario de référence suppose un maintien en l'état des caractéristiques propres à chaque région de leur régime de retraite par répartition : les pensions de retraite sont indexées sur le salaire net; l'âge de la retraite est fixé à 60 ans en Europe, 70 ans au Japon et 65 ans dans les autres zones ; le taux de remplacement du salaire net par la pension est de $75 \%$ en Europe, $40 \%$ au Japon, $30 \%$ aux États-Unis et $10 \%$ dans les autres zones.

Résultant de la combinaison de ces hypothèses, le taux d'intérêt mondial qui équilibre la demande et l'offre de capital dans l'ensemble des zones ne connaît pas un profil monotone au cours du XXI ${ }^{e}$ siècle (graphique 1) : pendant les trente premières années, il décroît régulièrement de 4,25 à $3,6 \%$, puis se redresse entre

1. Cette convergence résulte d'un double processus de rattrapage des rythmes annuels de croissance de la productivité et des niveaux vers ceux de la zone leader (Amérique du Nord). Le rythme tendanciel d'augmentation du progrès technique dans cette zone est une cible mouvante pour les autres. Il est supposé de $2 \%$ par an. Le processus est cependant lent dans la mesure où les niveaux de productivité des trois zones en développement sont à $20 \%$ environ du niveau de la zone leader en début du siècle et aux alentours de $40 \%$ à la fin (voir INGENUE [2000] pour plus de détails et de justifications sur ce choix). 


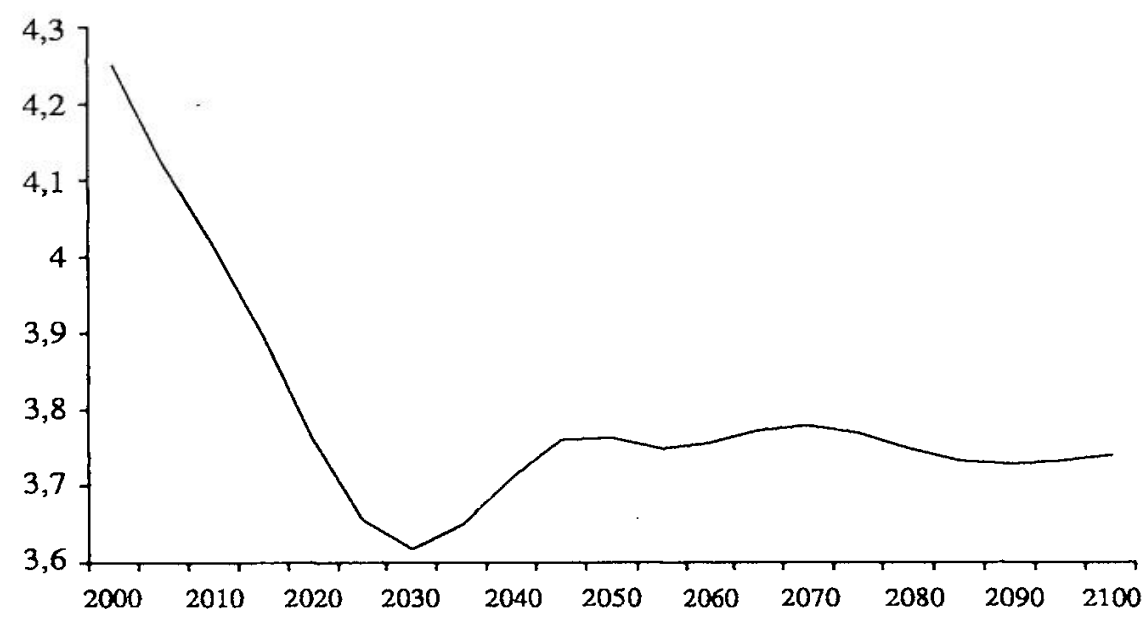

Graphique 1. Compte central, taux d'intérêt annuel mondial en \%

2030 et 2070 de 3,6 à $3,8 \%$, pour décroître lentement dans les deux dernières décennies. Une telle évolution ne résulte que peu des modifications de la demande de capital, qui est dominée par les mouvements démographiques, le rattrapage technologique restant mesuré : les populations actives de toutes les zones décélèrent jusqu'en 2025 , mais les zones à vieillissement démographique tardif ont des populations actives à la fois plus nombreuses et qui continuent à croître jusqu'en 2060 pour la plus avancée et en 2080 pour la moins avancée. La résultante de ces tendances est une demande de capital qui exerce une influence monotone décroissante sur le taux d'intérêt. Il faut donc se tourner du côté de l'offre de capital pour comprendre le retournement du taux mondial entre 2030 et 2050 .

Suivant l'hypothèse du cycle de vie, l'évolution heurtée de la structure démographique se transmet au profil du taux d'épargne agrégé des ménages. Or, l'Europe et le Japon sont les zones où la vague démographique est la plus prononcée : jusque vers 2025 , la population à forte épargne (tranche d'âge des 40 à 60 ans) croît, puis atteint une proportion culminante de la population totale de ces zones ; cette proportion décroît ensuite jusqu'en 2050. Cette évolution se reflète dans les taux d'épargne de ces zones (graphique 2) : élevés en 2000, ils baissent en Europe et au Japon jusqu'à atteindre un minimum en 2050. Au total, l'épargne mondiale augmente, mais à un rythme décroissant, dans le premier quart du $\mathrm{XXI}^{\mathrm{e}}$ siècle, puis connaît un ralentissement marqué jusqu'au milieu du siècle ; la demande d'investissement, quant à elle, reste soutenue ${ }^{1}$.

La répartition de la richesse au niveau mondial est mesurée par les taux de propriété du capital accumulé dans les zones. Ce rapport de la richesse privée d'une zone au stock de capital qui y est installé augmente avec la proportion des classes d'âge mûr dans la population active qui favorise l'épargne. Il diminue avec la hausse du taux de dépendance (ratio des inactifs aux actifs). Le stock

1. En raison du faible rythme de convergence technologique, les taux de croissance des PIB de zone ont des profils qui épousent largement ceux de la population active au début du siècle. Par la suite, les structures de population se stabilisant, les différences dans les rythmes de progrès technique, découlant du rattrapage même lent, deviennent prépondérantes. Les trois zones OCDE croissent à un taux de $2 \%$ par an à la fin du siècle. Les trois zones PVD à un taux proche de $3 \%$. 
Graphique 2. Compte central, taux d'épargne des ménages par région

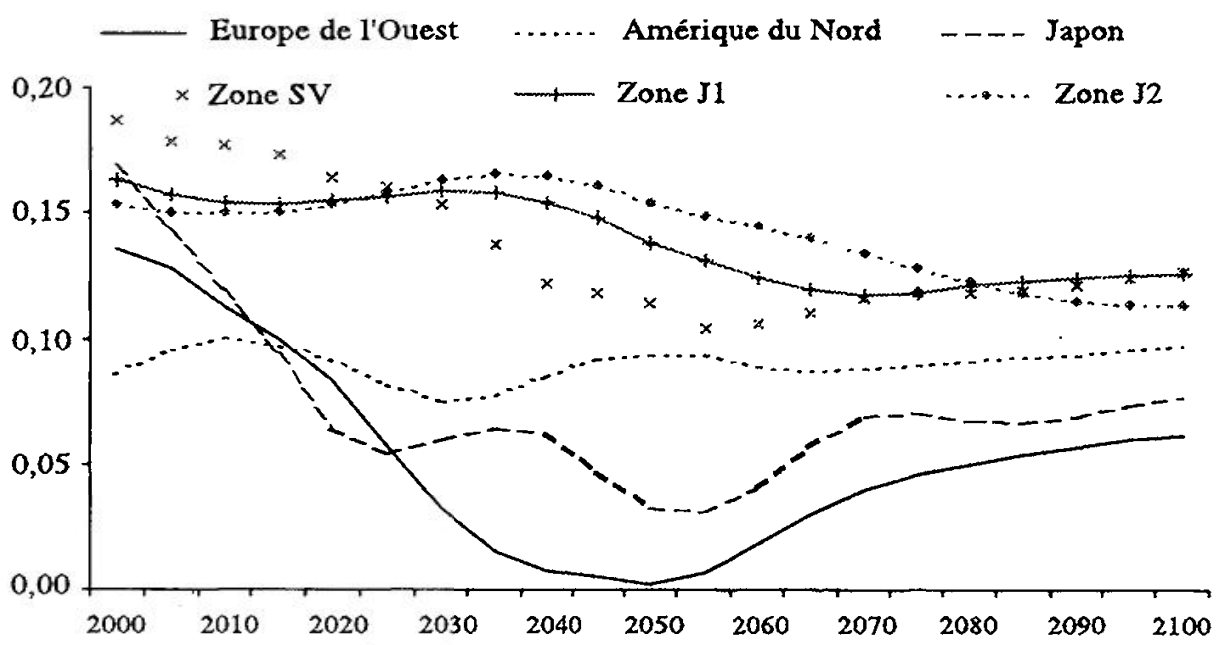

mondial de capital étant égal à la richesse mondiale, les taux de propriété sont interdépendants. Le taux d'intérêt d'équilibre ajuste la répartition de la richesse et le stock de capital. La combinaison de ces facteurs entraîne une profonde transformation de la richesse au cours du siècle (graphique 3a). L'Europe, le Japon et la «zone $S V$ » sont des régions créancières nettes dans les premières décennies, le poids des classes d'âge fortement épargnantes y augmentant. Corrélativement, les trois autres zones demeurent débitrices nettes pendant le premier demi-siècle. La répartition de la richesse commence à changer après 2030 , surtout au détriment de l'Europe : d'une position largement créancière dans les premières décennies, l'Europe basculerait dans une situation de plus en plus débitrice après 2040 ou 2050 jusqu'à devenir, en fin de siècle, la seule région débitrice.

\section{Graphique 3. Compte central}

a) Taux de propriété

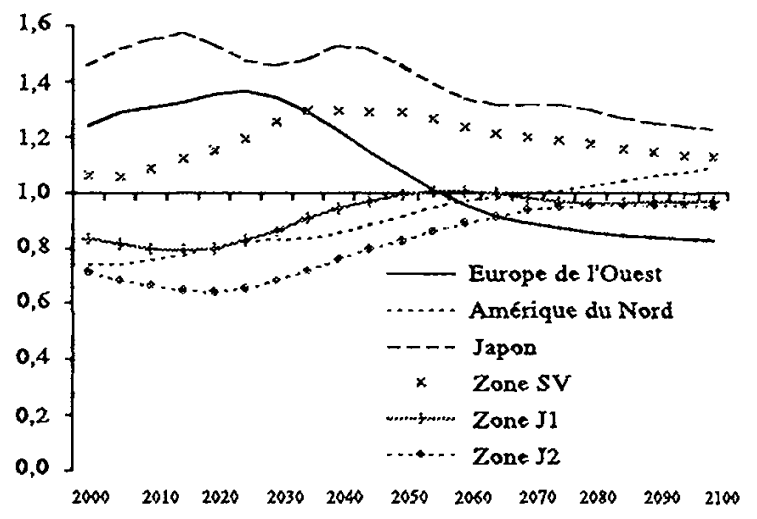

b) Taux de cotisation sociale

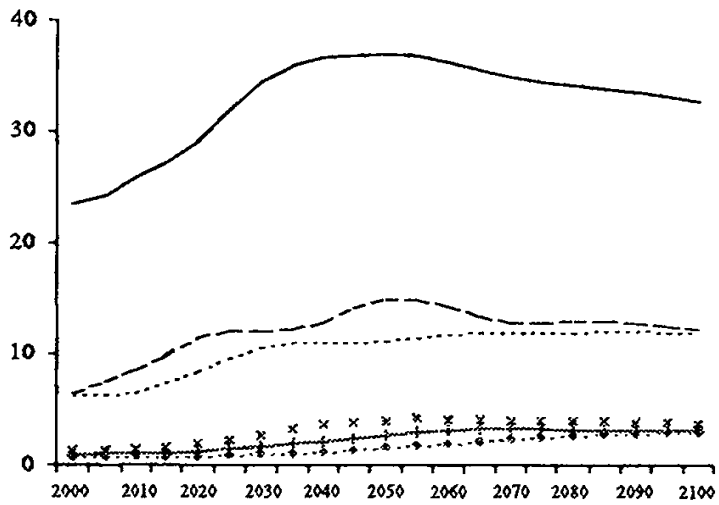

De fait, quelles que soient les hypothèses retenues sur les rythmes exogènes de croissance des productivités globales des facteurs dans les zones, la position extérieure de l'Europe se dégradera considérablement après 2030 (INGENUE [2001a]). La raison se trouve dans la combinaison des structures démographiques et des dispositifs institutionnels hérités du passé. L'onde de choc issue du baby boom fait basculer dans la retraite des effectifs nombreux, tandis que la 
population active se contracte relativement. Pendant plusieurs décennies, le taux d'épargne diminue. Du fait de régimes de retraite par répartition plus généreux qu'ailleurs, ces pressions sont plus importantes : ainsi les taux de cotisation moyens s'élèvent dans une proportion similaire à ceux des autres zones, mais les niveaux atteints en Europe au milieu du siècle sont sans commune mesure avec ceux des autres parties du monde (graphique $3 b$ ). Du fait de cette double tension sur les finances internes et externes, la question d'une réforme des régimes de retraite se pose de façon naturelle, même si en toute rigueur le mode de redistribution sociale des richesses entre les générations n'a pas vocation à se soucier de la solvabilité externe de la zone. En revanche, cette détérioration des positions financières de l'Europe se double d'un problème d'équité intergénérationnelle : le système de retraite est particulièrement favorable à ceux qui n'auront pas ou peu subi la hausse des taux de cotisation.

\section{CONSÉQUENCES MACROÉCONOMIQUES D'ÉVENTUELLES RÉFORMES DES RÉGIMES DE RETRAITE DE L'EUROPE}

Par rapport à cette situation de référence, trois réformes unilatérales des régimes de retraite par répartition de l'Europe sont étudiées. Bien que mises en cuvre, dans les simulations, à partir de l'an 2000, elles sont supposées être annoncées dès 1995.

1. Un maintien des taux de cotisation au niveau moyen où ils étaient à la fín $\mathrm{du} \mathrm{XX}^{\mathrm{e}}$ siècle. II en résulte une dégradation, progressive et très importante, du pouvoir d'achat des retraites par répartition qui est amputé de moitié entre 2000 et 2050. Cela entraîne une substitution partielle de la capitalisation à la répartition, car, pour se prémunir contre, ou tout au moins atténuer, cette baisse de leur niveau de vie futur, les individus en activité vont épargner davantage.

2. Un maintien des taux de remplacement avec un recul progressif de l'âge de cessation d'activité de 60 à 65 ans entre 2000 et 2020 . Cette réforme vise à élargir la taille de la population active et donc à accroître le volume de l'activité économique. La durée de la retraite étant réduite et la production plus élevée, la charge financière du vieillissement, dans sa phase la plus critique, est moins lourde et mieux partagée.

3. Enfin, une troisième variante illustre un tout autre phénomène. Il s'agit, au contraire, d'une pression accrue des retraités, dont le poids politique s'accroît, pour obtenir un partage social qui leur est plus favorable. Il se traduit ici par un changement du mode d'indexation des pensions, la politique s'exprimant comme un maintien des ratios de remplacement bruts.

L'effet direct des réformes concerne le taux d'épargne en Europe (graphique 4a). Le scénario de référence occupe une position centrale. Sans surprise, le taux d'épargne lui est toujours supérieur dans le scénario « de capitalisation» et toujours inférieur dans celui «d'extension de la protection sociale ». Parce qu'elles influencent fortement l'épargne en Europe, les variantes ont des incidences sur les autres zones du monde, via le taux d'intérêt. Pour ces deux premières réformes, comme les conditions de la demande de capital ne sont pas directement modifiées, le niveau du taux d'intérêt est, sur tout le siècle, 
plus bas dans le scénario de maintien des taux de cotisation et plus élevé dans le scénario d'indexation sur le salaire brut (graphique 4b).

\section{Graphique 4. Réformes des retraites de l'Europe}

a) Taux d'épargne des ménages européens

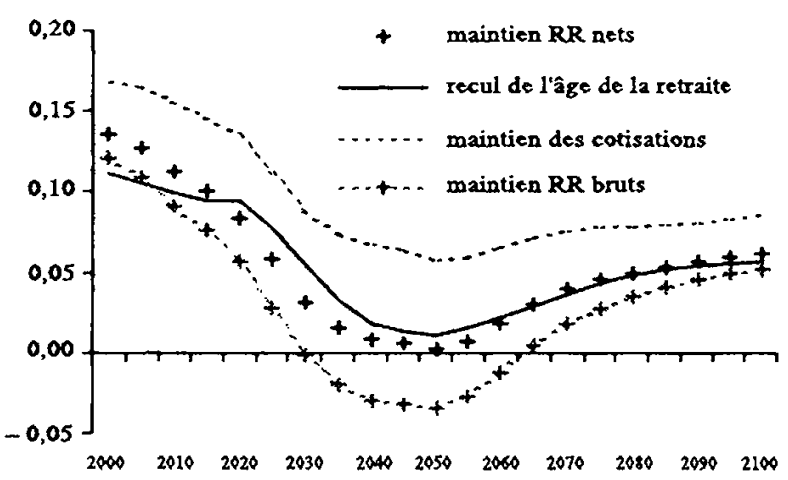

b) Taux d'intérêt mondial

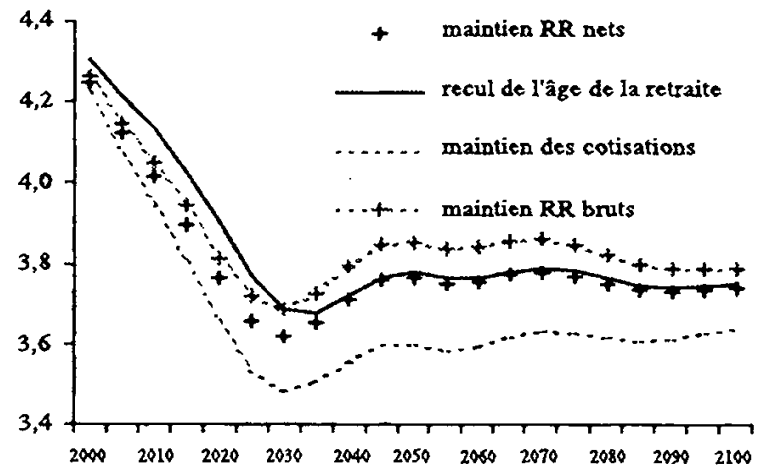

La comparaison avec le recul de l'âge de la retraite est plus délicate. Dès l'annonce de la réforme, le taux d'épargne tombe en dessous de celui de tous les autres scénarios : le recul de l'âge de la retraite augmente le revenu permanent des cohortes concernées (individus qui ont de 40 à 60 ans en 2000). La hausse anticipée du revenu se traduit par une augmentation immédiate de la consommation par tête. Mais dans les premiers temps de la mise en cuvre de la réforme, le nombre de retraités bouge peu, comme les salaires et les cotisations sociales, d'où une baisse instantanée de l'épargne. Par la suite, le taux d'épargne décroît moins vite que dans les autres scénarios pendant la durée de mise en œuvre de la réforme : la population active augmentant progressivement, une demande supplémentaire de capital se produit en Europe. C'est pourquoi le taux d'intérêt est plus élevé que dans tous les autres scénarios pendant la durée de réalisation de la réforme. Cet investissement plus élevé durant la période de montée en puissance de la réforme entraîne aussi une progression plus rapide des revenus d'activité par rapport au scénario de référence. La conjugaison des hausses de revenu et de taux d'intérêt pendant cette phase explique que le taux d'épargne décroisse moins vite que dans les autres scénarios jusqu'à 2020. Ensuite, le taux d'épargne est plus élevé que dans le scénario de référence puisque le poids de la population dont les revenus sont plus élevés, c'est-à-dire après le départ des enfants et jusqu'à la retraite, s'accrô̂t.

L'effet sur l'épargne des autres zones dépend essentiellement du profil de taux d'intérêt, et est donc similaire d'une zone à l'autre : plus il est haut, plus le taux d'épargne est élevé, cet effet induit s'estompe lentement après le milieu du siècle. En revanche, les variations des taux de propriété d'un scénario à l'autre dépendent pour les autres zones des écarts dans les taux de propriété en Europe dans la mesure où l'ensemble des zones sont liées par la contrainte d'égalité du capital et de la richesse dans le monde. Comme les taux de propriété varient beaucoup en Europe d'un scénario à l'autre, ces variations se refiètent en sens contraire pour l'ensemble des autres zones et généralement toutes du même côté. Sans surprise, l'augmentation de l'épargne dans le scénario de capitalisation est si forte que l'Europe reste en permanence créancière nette (graphique 5a). En revanche, le scénario d'indexation sur le salaire brut aggrave 
Graphique 5. Réformes des retraites de l'Europe

a) Taux de propriété

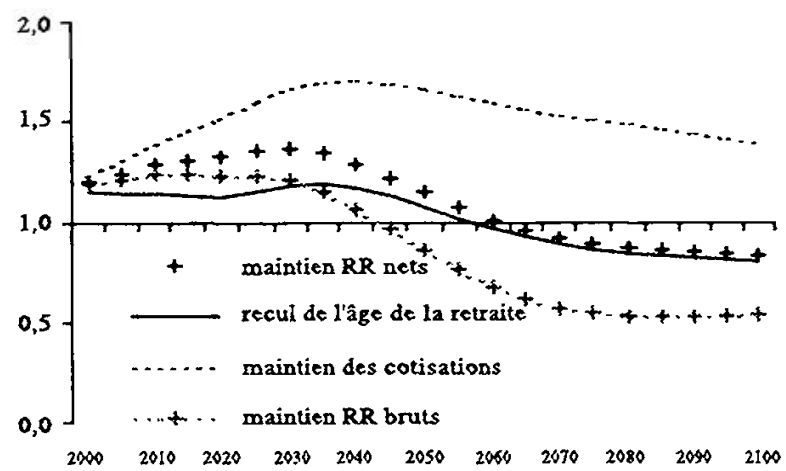

b) Taux de cotisation sociale

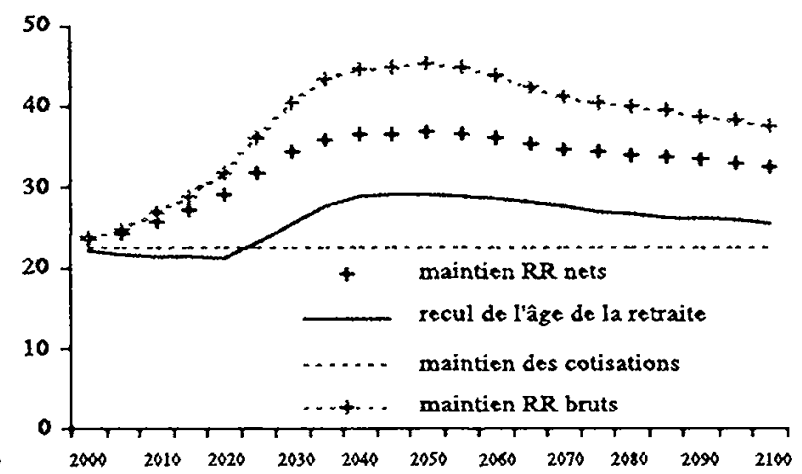

très sensiblement la détérioration des finances extérieures par rapport au scénario de référence. Quant au scénario d'allongement de la vie active, il réduit le taux de propriété à cause du fléchissement initial de l'épargne et de l'effort d'investissement supplémentaire au cours de la réalisation de la réforme. Ensuite, l'épargne accrue efface cet écart. Le profil du taux de propriété vient s'aligner sur celui du scénario de référence.

Mais le véritable critère de jugement de ces réformes sociales concerne le bien-être de la population et ne se trouve pas dans les ajustements financiers. Il faudrait pouvoir classer les différents scénarios de ce point de vue ainsi que de celui de l'équité intergénérationnelle. Avant d'aborder ce point dans la section suivante, concentrons-nous simplement ici sur la comparaison des situations en termes de niveaux de vie moyens à travers le monde afin d'évaluer et d'illustrer les interdépendances régionales.

Les deux scénarios de capitalisation et d'indexation sur les salaires bruts n'ont guère d'effet sur la croissance mondiale par rapport au scénario de référence. Aussi les effets sur la consommation par tête, en Europe d'un côté et dans le reste du monde de l'autre, sont-ils inversés (graphique 6). La capitalisation provoque un appauvrissement de la population européenne par rapport au scénario de référence jusqu'en 2040, un enrichissement ensuite. Le maintien des taux de remplacement avec indexation sur le salaire brut a un profil de consommation quasi symétrique. C'est la même disposition, mais inversée, que l'on trouve dans les autres zones. En effet, capitaliser dans des marchés financiers intégrés, c'est exporter des capitaux à l'étranger. Comme ce surcroît d'épargne européenne fait baisser le taux d'intérêt mondial, les pays bénéficiaires de cet apport peuvent investir plus. L'augmentation induite de la productivité soutient un niveau plus élevé de consommation par tête. Le processus s'inverse après la remontée du taux d'intérêt qui est favorable aux revenus du capital accumulé à l'étranger par les Européens.

Le scénario de recul de l'âge de la retraite est d'une nature différente, car il augmente la population active de l'Europe, et donc les dotations factorielles au niveau mondial. Il y a à la fois augmentation des salaires, de l'investissement et de l'épargne en Europe. Cette hausse des capacités productives de l'Europe élève la croissance mondiale, accroît le taux d'intérêt et donc incite les populations du reste du monde à placer leur épargne en Europe. Les profils de consommation par tête dans ce scénario résultent de ces processus. En Europe, 
Graphique 6. Réformes des retraites de l'Europe, consommations régionales par tête
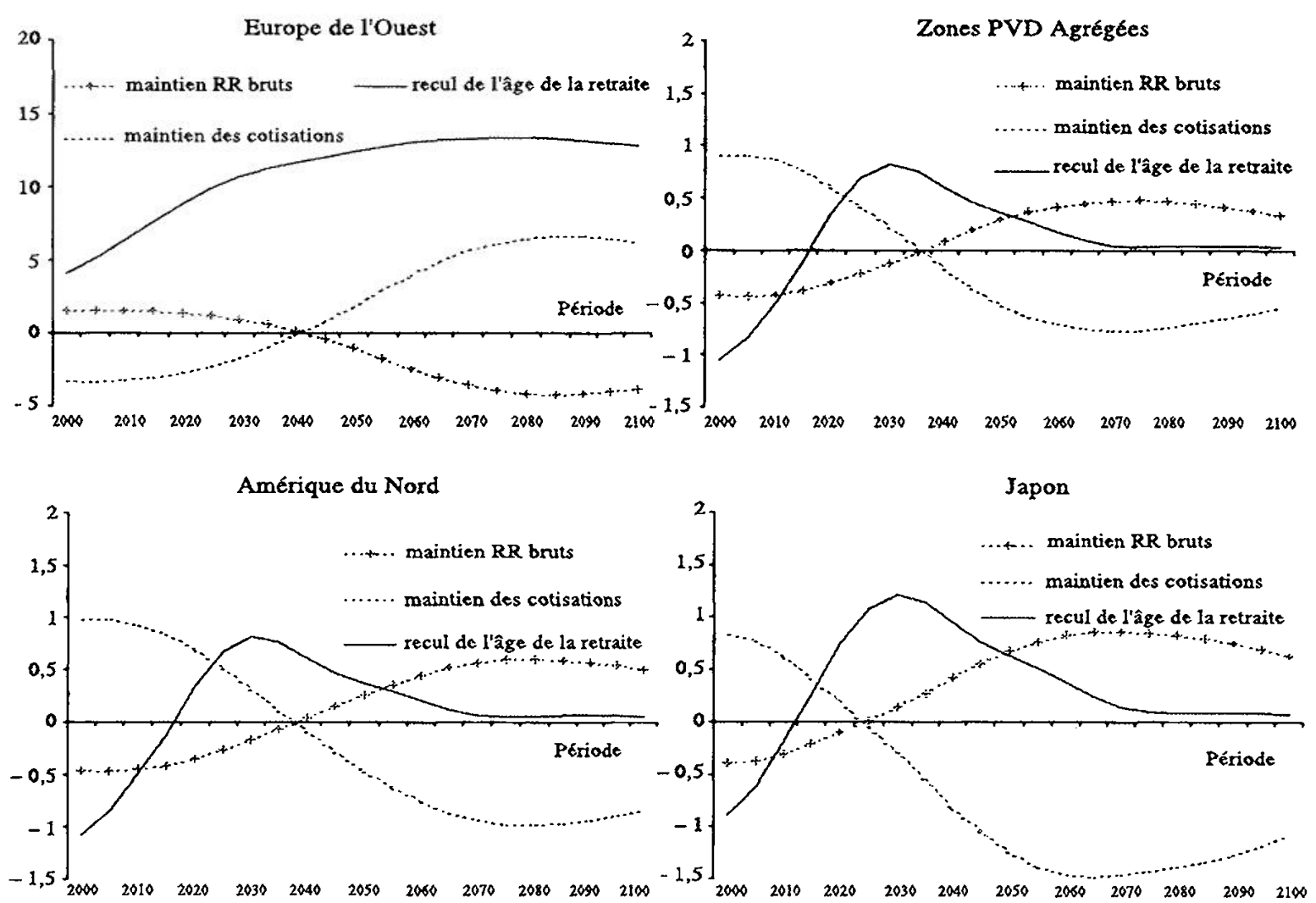

le niveau de consommation par tête est toujours au-dessus de celui de tous les autres scénarios. Dans les autres zones, on a d'abord une consommation plus faible qui découle du surcroît d'épargne exportée en Europe. Puis, la croissance mondiale plus forte disparaît après 2030 à partir d'un niveau plus élevé de produit par tête. Toutes les zones en bénéficient. Vers 2030, la consommation par tête est maximale. Puis le choc se résorbe progressivement. À la fin du siècle, la consommation hors Europe se retrouve au niveau qu'elle atteint dans le scénario de référence.

\section{INCIDENCES REDISTRIBUTIVE ET POLITIQUE DES RÉFORMES}

Réformer les systèmes de retraite répond en général à un besoin redistributif (devoir moral de compenser une éventuelle injustice entre les générations) mais aussi politique : elle doit être souhaitée ou approuvée par une majorité d'électeurs.

Dans cette dernière section, nous tenterons de dresser un bilan des réformes étudiées, en termes de partage des ressources entre les générations ainsi qu'en termes de solde électoral. Le bilan sur le partage des ressources consiste à identifier les générations gagnantes et les perdantes aux réformes, d'une part, et à voir dans quelle mesure la ré-allocation des richesses se rapproche d'un optimum social. Trois critères d'évaluation de l'impact des réformes sur les géné- 
rations sont retenus : un critère hédoniste, un critère financier et un critère de planificateur.

Le critère hédoniste est le niveau de bien-être atteint sur l'ensemble du cycle de vie pour chaque génération. Ce critère permet d'abord de déterminer quelles sont les générations qui bénéficient de ou au contraire perdent à la réforme ; il permet aussi de calculer un solde électoral.

Le critère financier est une évaluation actuarielle : il consiste à calculer le taux de rendement interne du système par répartition, c'est-à-dire le taux d'actualisation qui assure l'équilibre entre la somme actualisée des cotisations et la somme actualisée des pensions. Plus le rendement est grand, plus la générosité de la répartition est élevée. Cette propriété provient de l'antériorité des cotisations par rapport aux pensions. Les cotisations subissent en effet en moyenne un coefficient d'actualisation plus faible que les pensions qui sont perçues seulement à la fin du cycle de vie.

Le critère de planificateur par excellence est la somme actualisée des bienêtre de toutes les générations. Ce critère est intéressant mais demeure, en général, un problème majeur : quel facteur d'escompte faut-il utiliser ? Pour contourner ce problème, Touzé [1999a, 1999b] propose de calculer non pas la somme escomptée des bien-être mais le facteur d'escompte public que reproduit implicitement l'équilibre concurrentiel avec répartition, c'est-à-dire de déterminer la chronique de facteurs d'escompte qu'il faudrait implicitement utiliser pour que l'équilibre concurrentiel soit pleinement compatible avec le critère de justice intergénérationnel. L'évaluation des réformes revient alors à étudier les modifications induites dans les taux d'escompte implicites : si la chronique des facteurs après réforme est plus uniforme, elle est plus conforme à un critère de bien-être escompté qui appliquerait le même taux d'escompte à toutes les générations.

À ces trois critères, on pourrait penser ajouter un critère d'équité fiscale, une sorte de ratio à la Auerbach et al. [1991]. Ce critère est en effet une tentative pour mesurer l'écart apparent de taxation entre les générations présentes et les générations futures. Il n'est pas présenté ici pour la principale raison que dans le cadre des réformes étudiées, le maintien constant du taux de cotisation sociale est, par nature, une réforme qui garantit un traitement équitable des générations en matière de taux de prélèvement.

\section{Le critère du bien-être}

Le niveau de bien-être atteint par chaque génération est le critère naturel d'évaluation de 1'impact d'une réforme. Ce critère somme les niveaux d'utilité issus de la chronique des consommations sur l'ensemble du cycle de vie. Ces niveaux d'utilité sont pondérés par le facteur d'escompte privé. Cette mesure générale tient donc compte des préférences pour le présent et du caractère décroissant de l'utilité marginale. Dans le cadre du modèle à générations imbriquées dans sa version la plus simple, on sait que la fonction d'utilité indirecte - qui mesure le niveau de bien-être pour une législation donnée du système de retraite - est croissante des revenus du travail, du rendement du capital et du niveau des pensions, et décroissante du taux de cotisation. On sait également que, lorsque la dynamique de l'économie est efficiente, tout alourdissement du système par répartition est nécessairement préjudiciable à certaines généra- 
tions : même si le niveau des pensions augmente, la baisse de l'accumulation de patrimoine liée à l'accroissement des taux de cotisation (baisse du taux d'épargne) reste préjudiciable aux générations futures ${ }^{1}$.

Dans INGENUE, aucune utilité n'est accordée au loisir. Il en ressort que tout accroissement de l'offre de travail, via l'allongement de la durée d'activité légale, procure toujours une augmentation du bien-être par l'intermédiaire de la hausse résultante des revenus. La réforme d'allongement progressif de la durée d'activité est donc forcément Pareto-améliorante : elle augmente le bien-être de toutes les générations. En effet, l'augmentation de la durée d'activité apparaît comme une « bonne nouvelle » : elle s'assimile à un choc positif sur la productivité des travailleurs âgés qui n'étaient pas employés pour des raisons économiques (productivité supposée nulle). Cependant, si on admet que le travail des individus âgés peut avoir un coût moral élevé (travailler au-delà d'un certain âge paraît préjudiciable à la qualité de la vie), il faudrait en tenir compte dans les critères de bien-être. Avec la version actuelle d'INGENUE, ce coût n'est pas chiffré, ni chiffrable. Cela signifie qu'il est difficile d'apprécier le caractère bénéfique sur le bien-être des réformes d'allongement de la durée d'activité (cf. graphique 7).

Les deux autres réformes produisent des effets opposés. D'un côté, la réforme de «maintien du taux de cotisation » avantage les générations futures (celles nées à partir de 1985). D'un autre côté, la réforme «maintien des taux de remplacement bruts » avantage uniquement les générations actuelles (celles nées avant 1985). En effet, le vieillissement (processus d'allongement de la durée de la vie qui s'observe, dans INGENUE, jusqu'en 2050) et l'entrée en retraite des générations du baby-boom (phénomène massif pendant la période 2005-2015) devraient se traduire par une forte augmentation des taux de cotisation si les taux de remplacement sur les salaires bruts étaient maintenus constants (cf. graphique 3b) ou par une réduction drastique de ces mêmes taux de remplacement si le taux de cotisation était fixé. La première option « maintien des taux de remplacement bruts » est avantageuse pour les générations actuelles (cf. graphique 7) puisqu'elle reporte le fardeau de la transition démographique sur les générations futures (taux élevé de cotisation et faible niveau d'accumulation de patrimoine). À l'inverse, la seconde option «maintien des taux de cotisation » fait subir l'essentiel du poids de la transition démographique aux générations actuelles, et libère les générations futures de ce fardeau, puisque 1'accumulation de capital résultante est plus grande ${ }^{2}$ (cf. graphique 7).

En réalisant le décompte à chaque période des gagnants aux réformes, on peut calculer un taux de satisfaction sous la forme d'une moyenne pondérée par l'effectif de chaque cohorte. Pour l'année de la réforme, à savoir 1995, ce taux de satisfaction peut s'assimiler à un solde électoral qui traduit le soutien politique des réformes en cas de soumission à un vote. L'évaluation temporelle de

1. Les réformes des systèmes de retraite en Europe modifient également le bien-être dans les autres régions du monde puisqu'elles influencent les offres et demandes sur le marché mondial du capital, donc le taux d'intérêt mondial et les salaires régionaux.

2. Ces résultats sont conditionnés au caractère efficient de la dynamique : il n'est pas possible d'augmenter le bien-être d'une génération sans réduire le bien-être d'autres générations. 
ce taux traduit, dans une certaine mesure, l'approbation politique des générations futures ${ }^{1}$. Le graphique 8 reproduit l'évolution du taux d'approbation associé aux deux réformes qui garantissent un « maintien des taux de remplacement bruts » ou «maintien du taux de cotisation sociale ». En ce qui concerne la réforme " recul de l'âge de la retraite », vu son caractère Pareto-améliorant, le taux d'approbation est trivialement égal à $100 \%$.

La réforme «maintien du taux de remplacement brut » étant favorable aux générations présentes, il se trouve que cette réforme recueille l'unanimité des suffrages jusqu'en 2000. À partir de 2005, la génération née en 1985-1989 entre dans sa majorité. On observe alors une chute régulière du taux de satisfaction (ou approbation). La réforme n'est plus approuvée à partir de 2040. Une évolution opposée s'observe dans le cadre de la réforme de « maintien du taux de cotisation sociale ».
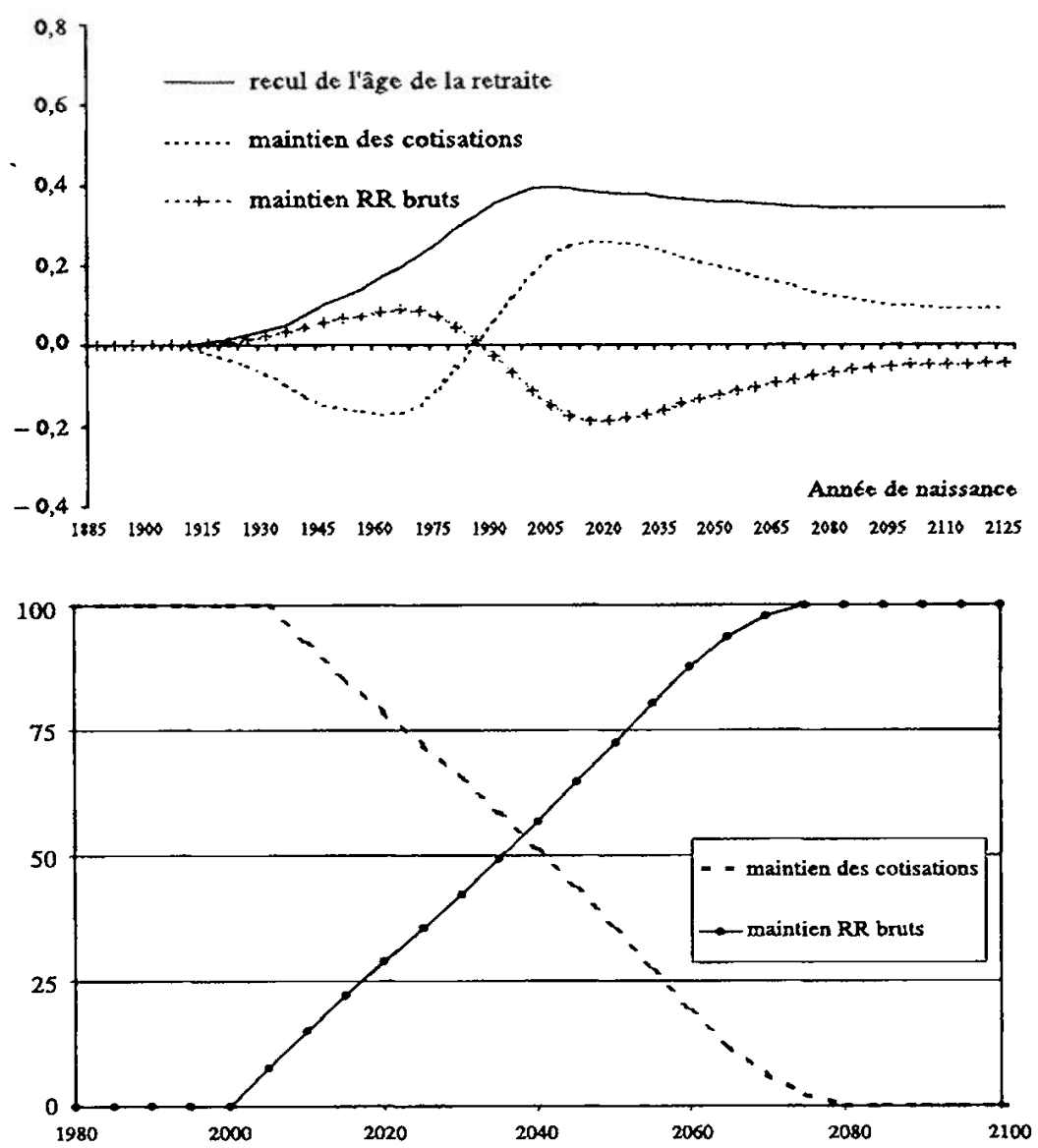

Graphique 7.

Variations relatives du bien-être (en \%)
Graphique 8. Taux de satisfaction (en \%)

\section{Un critère financier}

Un critère financier d'évaluation de la performance d'un système de retraite par répartition est le taux de rendement interne. Ce critère mesure précisément le taux d'actualisation qui égalise la valeur actualisée des cotisations à celle des

1. L'approbation par les générations futures d'un projet de réforme soulève des problèmes complexes de cohérence temporelle et de jeux dynamiques que nous n'abordons pas ici. 
pensions de retraite. Le taux de rendement interne est influencé par la générosité de la répartition : des pensions plus élevées ou des cotisations moindres influencent positivement le taux de rendement. Mais du point de vue de l'équilibre comptable des finances du système de retraite, l'évolution du montant des pensions est connectée à celle du taux de cotisation : une hausse des pensions se traduit mécaniquement par une hausse des cotisations. Ces hausses sont donc inégalement partagées entre les générations, ce qui explique l'existence de gains différenciés selon les générations pour l'ensemble des réformes simulées.

Les trois réformes considérées modifient la générosité de la répartition. En 1995, les générations les plus âgées sont les plus concernées par une politique qui modifie les droits à la retraite. Une hausse des pensions, ce qui est le cas avec la réforme « maintien des taux de remplacement bruts », leur est particulièrement favorable, car l'essentiel des hausses du taux de cotisation concerne les générations futures. Pour les générations nées à partir de 1995, le taux de rendement interne diminue, tandis qu'il augmente pour les autres générations.

À l'inverse, une baisse du montant des pensions, par un maintien constant du taux de cotisation, apparaît préjudiciable pour les générations les plus anciennes et procure une amélioration du taux de rendement uniquement pour les générations nées après 1995 .

Si la durée d'activité augmente, la période de cotisation s'accrôit au détriment de la période de pensions : de façon mécanique, le taux de rendement diminue. Mais, à plus long terme, la baisse des taux de cotisation, que permet de réaliser 1'allongement de la durée d'activité, conduit à une amélioration du taux de rendement. Les générations qui subissent la réforme sont celles qui subissent principalement la réduction de la durée des pensions sans bénéficier de la baisse des cotisations. D'après nos simulations, le taux de rendement des générations nées après 1980 est plus élevé que celui du compte central.

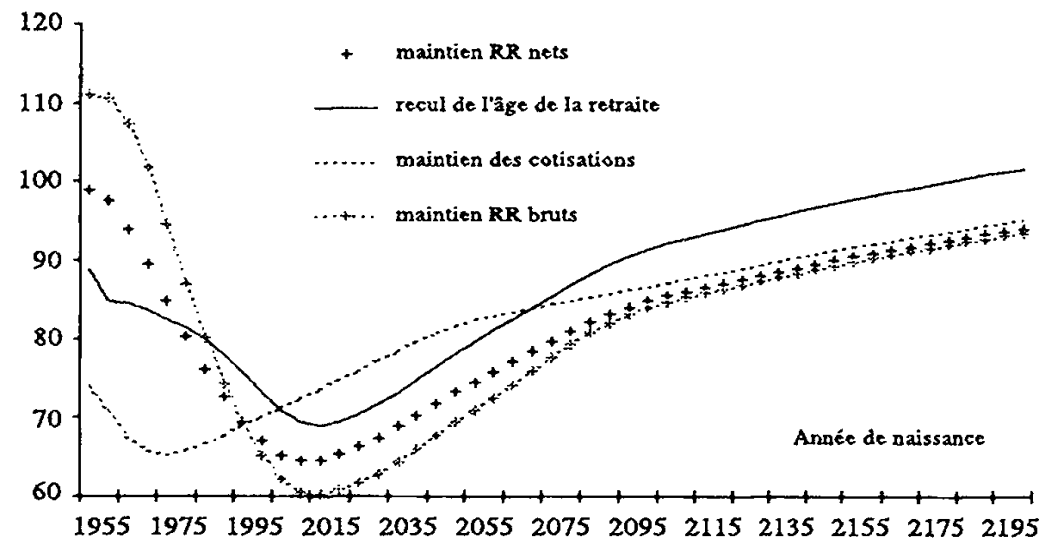

Graphique 9. Taux de rendement interne - Écart au compte central

Base $100=$ génération 1955 et scénario central

D'un point de vue théorique, la comparaison des variations des taux de rendement à celles du bien-être n'est pas évidente. Il n'y a aucun fondement pour justifier une parfaite concordance entre les deux. Néanmoins, il est intéressant de remarquer ici que les générations gagnantes (resp. perdantes) sont presque identiques (à l'exception des générations nées entre 1985 et 1994) quel que soit le critère utilisé en ce qui concerne les deux réformes « maintien des taux de remplacement bruts » et «maintien des taux de cotisation sociale ». En revanche, la réforme « recul de l'âge de la retraite » révèle des perdants d'un 
point de vue actuariel alors qu'il n'en existe pas selon le critère du bien-être. Ce résultat provient probablement du fait que, dans notre évaluation du taux de rendement, le supplément de revenu lié à l'accroissement de la durée d'activité n'est pas pris en compte. Si c'était le cas, il n'y aurait pas nécessairement de baisse du taux de rendement pour les premières générations.

\section{Un critère de planificateur : le facteur d'escompte public implicite (Touzé [1999a])}

La somme actualisée des bien-être de toutes les générations est un critère usuel de planification ${ }^{1}$. Deux raisons justifient l'escompte des bien-être : il faut que l'objectif de d'optimum social soit mesurable et donc que la somme des bien-être soit finie (ce n'est pas le cas si on n'escompte pas, à moins de maximiser un écart à un objectif stationnaire de bien-être: voir sur ce sujet les problèmes étudiés par Ramsey [1928] ; Koopmans [1965] ; Phelps [1965] ; Weitzman [1973] ; Michel [1993] ; Fleurbaey et Michel [1992 et 1994]); l'application d'un facteur d'escompte sur les bien-être peut refléter une préférence publique pour le présent. À l'optimum social, on observe la règle bien connue selon laquelle le planificateur égalise les utilités marginales, entre les générations présentes, qu'il pondère par le facteur d'escompte public. Selon ce critère, une réforme est socialement souhaitable si les pertes subies par certaines générations sont largement compensées par les gains d'autres générations.

La difficulté d'utilisation d'un critère de bien-être actualisés réside principalement dans le choix d'un facteur d'escompte (voir, par exemple, Chilchinisky [1996], pour une discussion sur le «bien-fondé » d'un facteur d'escompte). Touzé [1999a, 1999b] propose de contourner cette difficulté du choix d'un taux d'escompte en observant directement le taux d'escompte public implicite induit par l'équilibre de laissez-faire. Le raisonnement est le suivant : traiter avec impartialité toutes les générations, n'est-ce pas garantir la même valeur de facteur d'escompte? Dans ce contexte, une façon simple d'apprécier une réforme, qui modifie l'allocation des ressources dans le temps et entre les générations, est d'examiner comment se modifie la chronique des facteurs d'escompte: si la modification tend vers un alignement, cela signifie que le partage des ressources, que régule le système par répartition, se rapproche de celui qu'on observerait si un facteur d'escompte unique était appliqué à l'ensemble des générations, et inversement.

Par convention, on définit ici le facteur d'escompte, noté $\delta$, par le coefficient appliqué spécifiquement ${ }^{2}$ sur le bien-être d'une génération née à la date $t-c e$ facteur est inversement proportionnel au facteur d'actualisation - et on définit le facteur d'escompte total comme le produit des facteurs d'escompte implicites que l'on note $\Delta_{t}=\delta_{t} \cdot \Delta_{t-1}$. Ainsi, à l'instant 0 , la valeur du bien-être d'une génération née à la période $t$, que le planificateur prend en considération, est alors strictement égale à : $\Delta_{t} \frac{N_{t}}{N_{0}} V_{t}$ où $V_{t}$ est le niveau de bien-être effectif de la

1. Pour une discussion générale sur la justice entre les générations, on peut se reporter à l'article de Fleurbaey et Michel [1992].

2. On peut parler de facteur d'escompte marginal. 
génération née à l'instant $t$, et où $\mathrm{N}_{t}$ et $\mathrm{N}_{0}$ sont les tailles des générations respectivement nées à l'instant $t$ et à l'instant 0 . Le ratio démographique signifie que le planificateur accorde, en parallèle, un poids aux générations en fonction de leurs tailles relatives.

Dans le modèle INGENUE, pour une génération active à l'instant $t$, le taux d'escompte implicite se mesure exactement comme suit :

$$
\delta_{t}=\rho \cdot \frac{u^{\prime}\left(c_{t, 25 \text { ans }}\right)}{u^{\prime}\left(c_{t, 20 \text { ans }}\right)}=\rho \cdot\left(\frac{c_{t, 20 \text { ans }}}{c_{t, 25 \text { ans }}}\right)^{\frac{1}{\sigma}}
$$

où $\rho$ est le facteur d'escompte privé, $u$ la fonction d'utilité et $\sigma$ l'élasticité intertemporelle de substitution. Le taux d'escompte implicite apparaît comme un ratio des utilités marginales de deux générations successives concernant la même période de consommation ${ }^{1}$.

Les deux graphiques suivants représentent les facteurs d'escompte implicitement appliqués à chaque génération dans le cadre du modèle INGENUE. Le premier graphique présente le facteur spécifique $\delta_{t}$ et le second, le facteur implicite moyen $\left(\Delta_{t}\right)^{\frac{1}{t}}$. Les facteurs calculés correspondent à ceux visibles à partir de la mise en place des réformes, à savoir 1995. Le facteur moyen est évalué pour l'année de la réforme. Il correspond donc à la pondération moyenne que le planificateur accorde implicitement à chaque génération en 1995.

Le scénario du compte central fait apparaître une chute brutale du facteur d'escompte (qui reste néanmoins relativement faible) pour la génération née en 1960, puis une remontée très progressive vers un niveau supérieur mais à peu près équivalent à celui observé pour la génération 1955 : en termes de facteur d'escompte, les générations futures (celles qui naîtront après 2020-2025) semblent traitées de façon à peu près identique. Les variations du facteur d'escompte se situent entre + ou $-2 \%$ (en termes quinquennaux) du niveau de référence (compte central et génération 1955). Une réduction du facteur d'escompte traduit une augmentation de l'écart de consommation entre la génération considérée et celles qui la précèdent. À l'inverse, une hausse coïncide à une réduction de cet écart de consommation. Le facteur d'escompte moyen (moyenne géométrique observée en $1955^{2}$ ) radicalise les tendances tout en lissant un peu les évolutions du facteur d'escompte.

Les deux réformes, qui garantissent une législation constante sur les recettes ou sur les dépenses, ont des effets très clairs du point de vue du choix du partage des ressources entre les générations.

La réforme «maintien des taux de cotisation » se traduit par une élévation des facteurs d'escompte, modification qui induit concrètement plus d'épargne et plus de bien-être pour les générations futures. Pour les générations de la tran-

1. Ce critère est a priori peu sensible à l'hypothèse d'offre de travail exogène dans la mesure où les utilités retirées du loisir et de la consommation sont des fonctions séparables.

2. La génération 1955 est active en 1975. Les simulations sont réalisées à partir de cette date. Elle constitue une génération de référence assez naturelle car il s'agit de la première génération pour laquelle, on dispose d'une chronique complète de consommations sur l'ensemble du cycle de vie. 

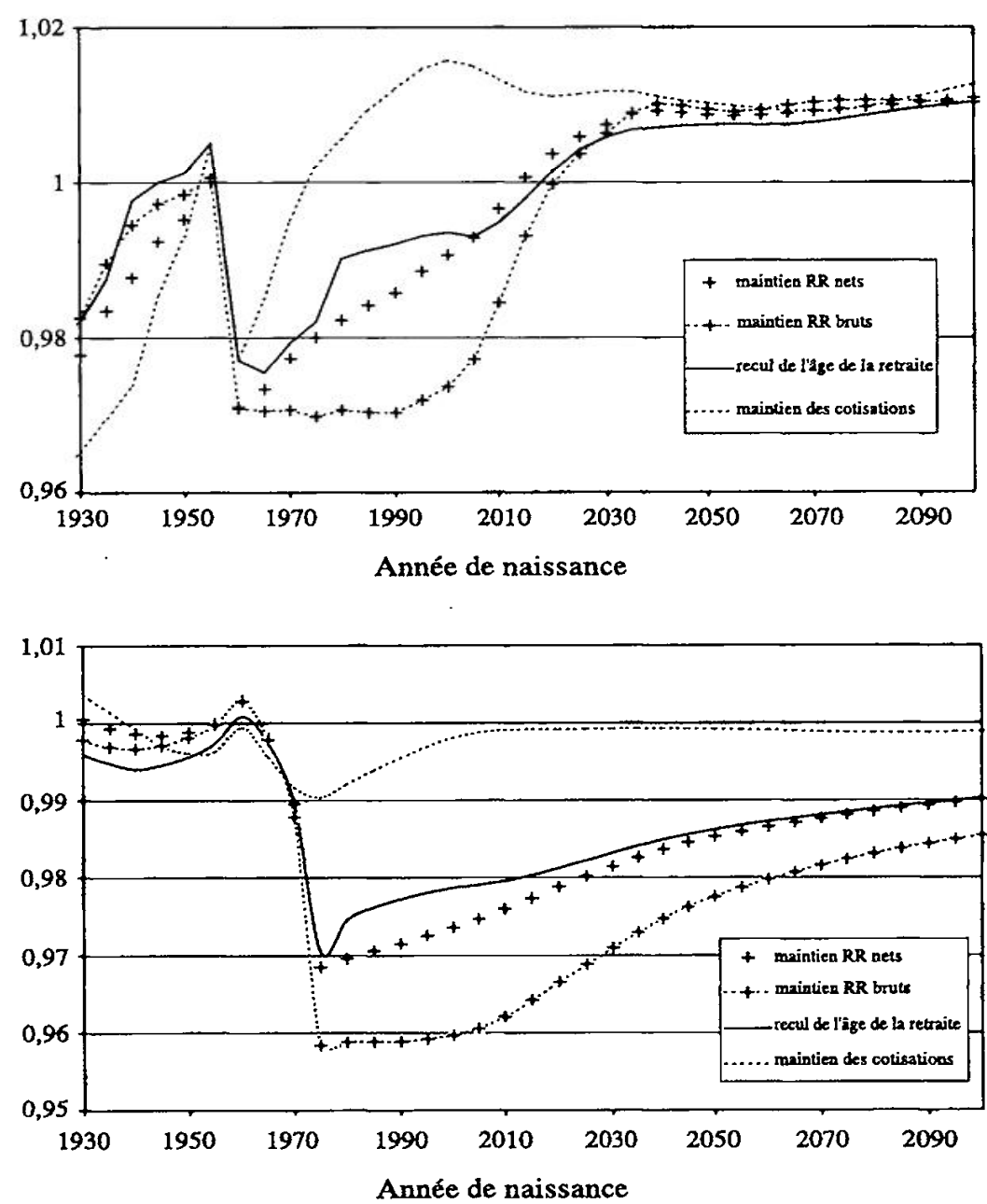

Graphique 10. Facteur d'escompte public implicite

Base $100=$ génération 1955 et compte de référence

\section{Graphique 11. Facteur d'escompte public implicite moyen Base 100 = génération 1955 et compte de référence moyenne géométrique par rapport}

à l'année de la mise en place de la réforme (1995)

sition, les effets demeurent contradictoires. D'un côté, leurs facteurs d'escompte augmentent : elles ont, certes, plus de poids. Mais, d'un autre côté, il y a aussi moins de ressources allouées à la consommation totale pour ces générations. C'est pourquoi, dans l'analyse de l'évolution des bien-être (cf. graphique 7), seules les générations nées après 1990 gagnent à cette réallocation. L'observation des moyennes géométriques des facteurs implicites révèle bien la meilleure prise en compte des jeunes et futures générations dans le partage courant de la richesse nationale (amélioration du ratio de consommation jeunes/vieux, accroissement du taux d'épargne favorable à plus de revenu dans le futur).

La réforme « maintien des taux de remplacement bruts » produit des effets opposés : plus de ressources sont réallouées à la consommation en général (et donc moins d'épargne), et à la consommation des générations les plus anciennes plus particulièrement. Cela se traduit par un important déplacement vers le bas du facteur d'escompte par rapport à sa trajectoire de référence. Les générations futures sont alors clairement pénalisées par la moindre épargne (cf. graphique 7 sur la variation du bien-être).

En revanche, la réforme de l'allongement de la durée d'activité a des effets plus difficiles à cerner en termes de modification de la chronique des facteurs d'escompte. L'augmentation du revenu national permet de réduire le ratio des consommations de la période courante concernant deux générations successives, et ce de façon durable. Mais, à long terme, une baisse très négligeable s'observe. 
En définitive, parmi les trois réformes étudiées, seul le maintien constant du taux de cotisation semble produire un réel alignement des facteurs d'escompte. Globalement, le supplément de richesse future, induit par cette réforme, et la moindre taxation produisent implicitement un traitement plus égal des générations au regard du critère de monotonie du taux d'escompte. En termes de taux d'escompte moyen, le résultat est très clair. D'une certaine manière, le choix de l'équité fiscale (taux de cotisation constant) est la réforme dont la solution se rapproche le plus de celle basée sur un choix de critère de somme de bien-être escomptés.

\section{CONCLUSION}

Cet article a présenté quatre scénarios d'évolution des finances sociales des systèmes de retraite en Europe : maintien du taux de remplacement brut, maintien du taux de cotisation, maintien du taux de remplacement net (scénario de référence) et recul de l'âge de la retraite. Le bilan des impacts des réformes a été entrepris sous trois aspects : économique, politique et redistributif.

D'un point de vue économique, la réforme d'alourdissement de la répartition (maintien du taux de remplacement brut) conduit à une réduction de la capacité d'épargne de l'Europe. À l'inverse, la réforme qui garantit une constance du poids des retraites (accroissement de la capitalisation) dans le PIB engendre un surcroît d'épargne. Quant à l'allongement de la durée d'activité, il a des effets contradictoires sur la capacité de financement de l'Europe : l'augmentation de la population active nécessite plus de capital ; 1'allègement de la retraite par répartition favorise un supplément d'épargne. Ces modifications de l'offre d'épargne européenne engendrent une variation du taux d'intérêt d'équilibre sur le marché du capital. Dans le cadre du modèle INGENUE, le marché du capital est un marché mondial. Cela se traduit par une amplitude de variation du taux d'intérêt beaucoup plus réduite que dans les modèles d'équilibre général en économie fermée, puisque l'offre d'épargne européenne est en partie diluée dans l'offre mondiale.

D'un point de vue redistributif, l'utilisation du critère du bien-être révèle que les générations nées avant 1985 gagnent, et sont donc politiquement favorables à un maintien des taux de remplacement sur le salaire brut, tandis que celles nées après 1985 sont gagnantes à une fixité du taux de cotisation. Elles sont donc politiquement favorables à un tel projet, mais elles n'obtiennent la majorité politique qu'en 2035. L'utilisation d'un critère financier de taux de rendement interne montre des résultats similaires. Enfin, l'examen des réformes avec un critère de planificateur montre que les réformes d'allongement de la durée d'activité ou de maintien constant du taux de cotisation semblent garantir l'application d'une chronique de facteurs d'escompte publics (implicites) plus homogène, et donc plus proche de la solution d'un problème de croissance optimale qui stipule un facteur d'escompte public constant. 


\section{RÉFÉRENCES BIBLIOGRAPHIQUES}

ANdo A. et Modigliani F. [1957], «Tests of the Life-Cycle Hypothesis of Saving: Comments and Suggestions », Bulletin of the Oxford Institute of Statistics.

Auerbach A. et Kotlikoff L. [1987], Dynamic Fiscal Policy, Cambridge, Cambridge, University Press.

AuErbach A., GoKhale J. et Kotlikoff L. [1991], «Generational Accounts : A Meaningful Alternative to Deficit Accounting », dans D. BRADFORD (ed.), Tax Policy and the Economy, vol. 5, Cambridge (Mass.), MIT Press, p. 55-110.

CAss D. [1972], « On Capital Accumulation in the Aggregative, Neoclassical Model of Economic Growth : A Complete Characterization », Journal of Economic Theory, 4, p. 200-223.

Cazes S., Chauveau T., Le CacheuX J. et Loufir R. [1992], « Démographie et retraites. $1^{\mathrm{xc}}$ partie : Le long terme », Revue de l'OFCE, 39, p. 93-149.

CHIlchINISKy G. [1996], «An Axiomatic Approach to Sustainable Development», Social Choice and Welfare, 13, p. 231-257.

CotTAVE R. [1991], Rapport de la «Mission Retraites », Paris, La Documentation française.

Dramond P. [1965], « National Debt in a Neoclassical Growth Model », American Economic Reviews, 55 (5).

FELDSTEIN M.S. [1974], « Social Security, Induce Retirement, and Aggregate Capital Accumulation ", American Economic Review, 86 (2).

Fleurbaey M. et Michel P. [1992], «Quelle justice pour les retraites ? », Revue d'économie financière, 23, p. 47-64.

Fleurbaey M. et Michel P. [1994], « Optimal Growth and Transfers between Generations », Recherches économiques de Louvain, 60 (3), p. 281-300.

INGENUE [2001a], "Vieillissement démographique et transferts internationaux d'épargne : premiers enseignements du modèle », Revue d'économie politique, avril, p. 195214.

INGENUE [2001b], « Macroeconomic Consequences of Pension Reforms in Europe : An Investigation with the INGENUE World Model », Working Paper CEPII-CEPREMAP-OFCE, décembre.

KoOPMans T.C. [1965], « On the Concept of Optimal Economic Growth », in The Econometric Approach to Development Planing, Pontificae Academiae Scientiarum Scripta Varia, 28, p. 225-287.

Kotlikoff L.J. [1996], «Privatization of Social Security at Home and Abroad », American Economic Review, 86 (2).

LE CACHEux J. et TOUZÉ V. [2002], «Les modèles d'équilibre général calculable à générations imbriquées : enjeux, méthodes et résultats ", Revue de l'OFCE, 80, p. 87113.

Malinvaud E. [1953], « Capital Accumulation and Efficient Allocation of Resources », Econometrica, 21, p. 223-268.

MrCHEL P. [1993], « Croissance et équilibre intertemporel : une présentation simple d'un modèle de base ", dans P. MALGRANGe et SALVAS-BRonsard L. (ed.), Macroéconomie : développements récents, Economica-Presses de l'Université du Québec.

Modigliani F. [1986], «Life Cycle, Individual Thrift, and the Wealth of Nations », Nobel Lecture, American Economic Review, 76 (3).

OBSTFELD M. et ROGOFF K. [1996], Foundations of International Macroeconomics, Cambridge, MIT Press. 
Phelps E.S. [1965], « Second Essay on the Golden Rule of Accumulation », American Economic Review, 55, p. 793-814.

RAMSEY F. [1928], « A Mathematical Theory of Saving », Economic Journal, 39, p. 543559.

Solow R. [1956], «A Contribution to the Theory of Economic Growth », Quarterly Journal of Economics, 70, p. 65-94.

TOuZE V. [1999a], Financement de la sécurité sociale et équilibre entre les générations, thèse de doctorat, Université Paris $X$.

Touzté V. [1999b], « Réforme de la sécurité sociale : quelques enseignements des modèles à générations imbriquées », document de travail, CREST et THEMA.

WertzMan M.L. [1973], « Duality Theory for Infinite Horizon Convex Models », Management Science, 19, p. 783-789. 